\title{
Multi-Target Prediction of Neuroprotective Drugs, Synthesis, Assay, and Theoretical Study of Rasagiline Carbamates
}

\author{
Francisco J Romero Durán ${ }^{1}$, Nerea Alonso ${ }^{1}$, Olga Caamaño ${ }^{1}$, Xerardo García-Mera ${ }^{1, *}$, Matilde \\ Yañez ${ }^{2}$
}

1 Department of Organic Chemistry, Faculty of Pharmacy, University of Santiago de Compostela (USC), 15782, Santiago de Compostela, Spain.

2 Department of Pharmacology, USC, 15782, Santiago de Compostela, Spain.

Published: 4 December 2015

\begin{abstract}
In this work, we developed a multi-target model for neuroprotective compounds reported in the CHEMBL database. The model predicted correctly $>8300$ experimental outcomes with Accuracy, Specificity, and Sensitivity above $80-90 \%$ in training and external validation series. This is model can different outcomes for $>30$ experimental measures in $>400$ different experimental protocols and related to $>150$ molecular and cellular targets present in 11 different organisms (including human). After that, we reported by the first time, the synthesis, characterization, and experimental assays of new series of chiral 1,2-rasagiline carbamate derivatives; not reported in previous works. This work is a synopsis of the results presented in our previous paper: Int J Mol Sci. 2014 Sep 24;15(9):17035-64. doi: 10.3390/ijms150917035.
\end{abstract}

Keywords: CHEMBL; Neuroprotective agents; Rasagiline derivatives; Asymmetric synthesis

* Corresponding authors: X García-Mera (xerardo.garcía@usc.es)

\section{Introduction}

The discovery of new drugs for the treatment of neurodegenerative diseases such as Alzheimer's, Parkison's, and Huntington's diseases, Friedreich ataxia and others an important goals of medicinal chemistry [1-4]. In order to develop such computational models we need to use modeling techniques to process chemical information from public databases. These databases have accumulated immense datasets of experimental results of pharmacological trials for many compounds. For instance, CHEMBL[5, 6], https://www.ebi.ac.uk/chembldb, is one of the biggest with more than $11,420,000$ activity data 
for $>1,295,500$ compounds, and 9,844 targets. In our previous work, we reported the first multitarget, multi-output, and multi-scale ALMA model for CHEMBL data of neuroprotective / neuro-toxic effect of drugs. After that, we reported by the first time, the synthesis, characterization, and experimental assays of new series rasagiline carbamate derivatives; not reported in previous works.

\section{RESULTS AND DISCUSSION}

\subsection{Development of New Model for}

Prediction of Drug-Target Networks

Model training and validation. We report a model to predict when the $i^{\text {th }}$ compound may present a high $\left(\mathrm{L}_{\mathrm{ij}}\left(\mathrm{c}_{\mathrm{q}}\right)=1\right)$ or not $\left(\mathrm{L}_{\mathrm{ij}}\left(\mathrm{c}_{\mathrm{q}}\right)=0\right)$ value of the experimental parameter used to characterize interaction with a molecular or cellular target involved in neuroprotective/neurodegenerative process. The output $\mathrm{S}_{\mathrm{ij}}\left(\mathrm{c}_{\mathrm{q}}\right)$ of our multi-output model depend on both chemical structure of the $i^{\text {th }}$ drug $d_{i}$ and the set of conditions selected to perform the biological assay $\left(\mathrm{c}_{\mathrm{q}}\right)$ including the $\mathrm{j}^{\text {th }}$ target, of course. In consonance, the ALMA model should predict different probabilities if we change the organisms ( $\left.\mathrm{c}_{1}\right)$, the biological assays $\left(\mathrm{c}_{2}\right)$, the molecular / cellular target $\left(c_{3}\right)$, or the standard experimental parameter measured ( $\left.c_{4}\right)$, for the same compound [7]. The best ALMA-entropy model found in this work was:

$$
\begin{gathered}
S_{i j}\left(c_{q}\right)=1.1396-0.4039 \cdot p\left(c_{l}\right) ? \theta_{1}^{i}+0.1993 \cdot \Delta \theta_{1}^{i}\left(s_{x}\right)+0.4349 \cdot \Delta \theta_{1}^{i}\left(a_{u}\right) \\
-0.0202 \cdot \Delta \theta_{1}^{i}\left(o_{t}\right)-0.0017 \cdot \Delta \theta_{1}^{i}\left(t_{e}\right) \\
N=2661 \quad R_{c}=0.72 \quad \chi^{2}=1913.007 \quad p<0.005
\end{gathered}
$$

The statistical parameters for the above equation in training are: Number of cases used to train the model (N), Canonical Regression Coefficient (Rc), Chi-square $\left(\chi^{2}\right)$, and p-level [8]. The probability cut-off for this LDA model is ${ }^{i} p_{1}\left(c_{q}\right)$ $>0.5 \Rightarrow \mathrm{L}_{\mathrm{ij}}\left(\mathrm{c}_{\mathrm{q}}\right)=1$. It means that the drug $\mathrm{d}_{\mathrm{i}}$ predicted by the model with probability $>0.5$ are expected to give a positive outcome in the $\mathrm{q}^{\text {th }}$ assays carry out under the given set of conditions $\mathrm{c}_{\mathrm{q}}$. This ALMA-entropy model present excellent performance in both training and external validation series with Sensitivity (Sn), Specificity (Sp), and Accuracy (Ac) $>80 \%$. Values higher than $75 \%$ are acceptable for LDAQSAR models, according to previous reports [913].

\subsection{Experimental and Theoretical Study of New Compounds}

Synthesis and experimental assay of new 1,2rasagiline derivatives. The compounds $2,3,4$, $5,6,7,8$ and 9 were synthesized according to the strategy given in Figure 2. As shown in this scheme, they were synthesized from the aminoalcohol 1 [(1R,2S)-(+)-1-amino-2-indanol], a commercial product. The alkylation of 1 with propargyl bromide and potassium carbonate in hot acetonitrile provided, in a global yield of $92 \%$, a mixture of the corresponding mono- and dipropargylated derivatives (2 and $\mathbf{3}$ ), which were separated by flash column chromatography using hexane/EtOAc (3:1) as eluent. Compound 3 was converted to the corresponding acetate (4) and benzoate (5) by treatment with acetic anhydride or benzoyl chloride, $\mathrm{Et}_{3} \mathrm{~N}$ and catalytic amounts of DMAP in MeCN. The carbamate derivatives $(\mathbf{6}, \mathbf{7}, \mathbf{8}$ and 9) were synthesized, from the hidroxy mono- or dipropargylaminoindans ( 2 and $\mathbf{3}$ ), by reaction with the corresponding dialkylcarbamyl chloride in $\mathrm{NaH}$ and acetonitrile following the procedure described in the literature [14]. 


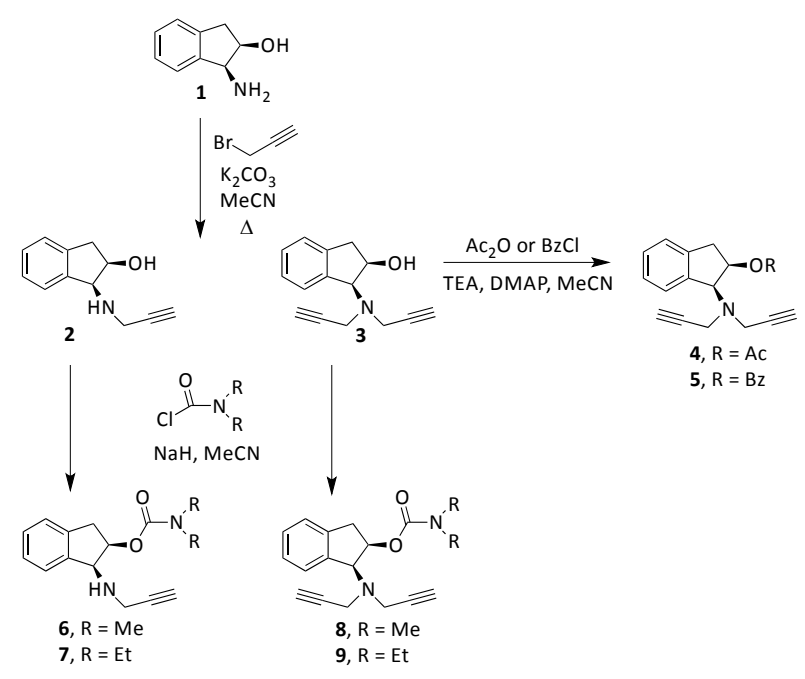

Figure 1. Synthesis of compounds 2-9

The new compounds synthesized in this work (2, $3,4,5,6,7,8$, and 9) were subjected to an initial study to determinate its neuroprotective ability in both the presence and the absence of neurotoxic agents (ANA). The method of reduction of the 3(4,5-Dimethylthiazol-2-yl)-2,5-

diphenyltetrazolium bromide (MTT) was used to ascertain the cell viability, given by the number of cells present in the culture. The ability of cells to reduce MTT is an indicator of the integrity of mitochondria, and its functional activity is interpreted as a measure of cell viability [15]. Three assays were conducted in a culture of motor cortex neurons of 19-day-old SpragueDawley rat embryos. Firstly, we studied the ability to induce a neuroprotective effect in the absence of any neurotoxic stimulation. Secondly, we studied the neuroprotective effect in the presence of glutamate, a compound that causes a pathological process in which neurons are damaged leading to apoptosis when its receptors such as the NMDA and AMPA are overactivated. Lastly, the ability of the compounds synthesized to protect from damage by $\mathrm{H}_{2} \mathrm{O}_{2}$, that causes neuronal death by Oxidative stress, was analyzed. The results obtained allow to deduce the existence of a moderate neuroprotective effect in the absence of any toxic stimulus, presenting the best results type $\mathbf{6}$ and 9 carbamate derivatives, with values of $11.5 \%$ and $8.4 \%$ respectively, followed by the compound $\mathbf{3}$, 4, and 7 with values slightly above $4 \%$ (see Figure 2).

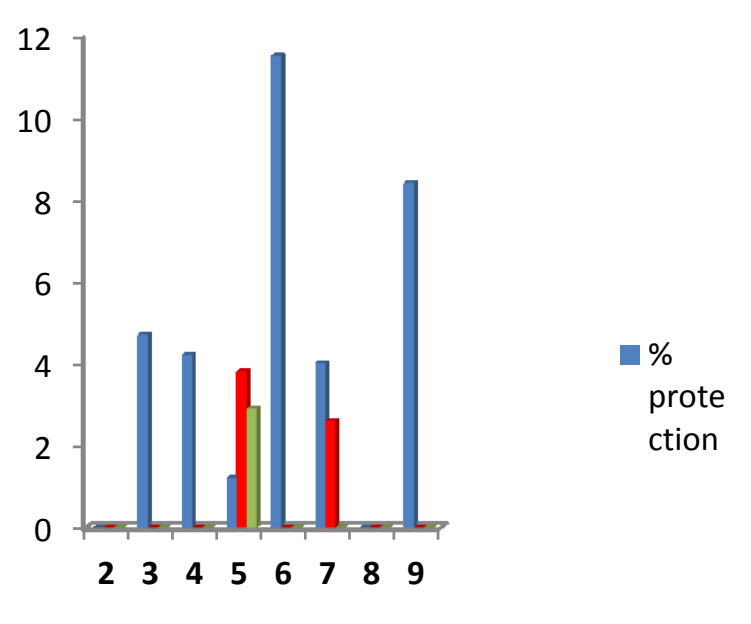

Figure 2. Results of the experimental assay of Neuroprotective effect of the new compounds. Predict new drugs in other assays. We used the ALMA-entropy model to predict the more probable results for all the new rasagiline derivatives, synthesized in this work, in $>500$ assays not carried out experimentally. When the molecular descriptors (entropy indices) of the new rasagiline derivatives were introduced in our model we obtained the probable interaction with different targets. The model predicts that most of them could interact with the subunits A and B of the 5-hidroxy-tryptamine type 3 receptors (5HT3Rs). These results seem to be consistent with the literature, since the antagonists of 5-HT3Rs have been related to neuroprotective properties in vitro and in vivo [16].

\section{Acknowledgements}


The authors thank the Xunta de Galicia for financial support of this work under project 07CSA008203PR.

\section{Conflict of Interest}

The authors declare no conflict of interest

\section{References}

1. Allegri RF, Guekht A: Cerebrolysin improves symptoms and delays progression in patients with Alzheimer's disease and vascular dementia. In: Drugs Today (Barc). vol. 48 Suppl A. United States: 2012 Prous Science, S.A.U. or its licensors; 2012: 25-41.

2. $\quad$ Park NH: Parkinson disease. JAAPA 2012, 25(5):73-74.

3. Morris HR, Waite AJ, Williams NM, Neal JW, Blake DJ: Recent advances in the genetics of the ALS-FTLD complex. Curr Neurol Neurosci Rep 2012, 12(3):243-250.

4. Trushina E, McMurray $\mathrm{CT}$ : Oxidative stress and mitochondrial dysfunction in neurodegenerative diseases. In: Neuroscience. vol. 145. United States; 2007: 1233-1248.

5. Heikamp K, Bajorath J: Large-scale similarity search profiling of ChEMBL compound data sets. $J$ Chem Inf Model 2011, 51(8):1831-1839.

6. Gaulton A, Bellis LJ, Bento AP, Chambers J, Davies M, Hersey A, Light Y, McGlinchey S, Michalovich D, Al-Lazikani B et al: ChEMBL: a large-scale bioactivity database for drug discovery. Nucleic Acids Res 2012, 40(Database issue):D1100-1107.

7. Gerets HH, Dhalluin S, Atienzar FA: Multiplexing cell viability assays. Methods Mol Biol 2011, 740:91-101.

8. Hill T, Lewicki P: STATISTICS Methods and Applications. A Comprehensive Reference for Science, Industry and Data Mining, vol. 1. Tulsa: StatSoft; 2006

9. Patankar SJ, Jurs PC: Classification of inhibitors of protein tyrosine phosphatase 1B using molecular structure based descriptors. J Chem Inf Comput Sci 2003, 43(3):885-899.

10. Garcia-Garcia A, Galvez J, de Julian-Ortiz JV, Garcia-Domenech R, Munoz C, Guna R, Borras R: New agents active against Mycobacterium avium complex selected by molecular topology: a virtual screening method. $J$ Antimicrob Chemother 2004, 53(1):65-73.

11. Marrero-Ponce Y, Castillo-Garit JA, Olazabal E, Serrano HS, Morales A, Castanedo N, Ibarra-Velarde F, Huesca-Guillen A, Sanchez AM, Torrens F et al: Atom, atom-type and total molecular linear indices as a promising approach for bioorganic and medicinal chemistry: theoretical and experimental assessment of a novel method for virtual screening and rational design of new lead anthelmintic. Bioorg Med Chem 2005, 13(4):1005-1020.

12. Casanola-Martin GM, Marrero-Ponce Y, Khan MT, Ather A, Sultan S, Torrens F, Rotondo R: TOMOCOMD-CARDD descriptors-based virtual screening of tyrosinase inhibitors: evaluation of different classification model combinations using bond-based linear indices. Bioorg Med Chem 2007, 15(3):1483-1503.

13. Casanola-Martin GM, Marrero-Ponce Y, Khan MT, Khan SB, Torrens F, Perez-Jimenez F, Rescigno A, Abad C: Bond-based 2D quadratic fingerprints in QSAR studies: virtual and in vitro tyrosinase inhibitory activity elucidation. Chemical biology \& drug design 2010, 76(6):538-545. 
14. Sterling J, Herzig Y, Goren T, Finkelstein N, Lerner D, Goldenberg W, Miskolczi I, Molnar S, Rantal F, Tamas T et al: Novel dual inhibitors of AChE and MAO derived from hydroxy aminoindan and phenethylamine as potential treatment for Alzheimer's disease. In: J Med Chem. vol. 45. United States; 2002: 5260-5279.

15. Mosmann $\mathrm{T}$ : Rapid colorimetric assay for cellular growth and survival: application to proliferation and cytotoxicity assays. In: J Immunol Methods. vol. 65. Netherlands; 1983: 55-63.

16. Fakhfouri G, Rahimian R, Ghia JE, Khan WI, Dehpour AR: Impact of 5-HT(3) receptor antagonists on peripheral and central diseases. Drug Discov Today 2012, 17(1314):741-747.

(C) 2015 by the authors; licensee MDPI, Basel, Switzerland. This article is an open access article distributed under the terms and conditions defined by MDPI AG, the publisher of the Sciforum.net platform. Sciforum papers authors the copyright to their scholarly works. Hence, by submitting a paper to this conference, you retain the copyright, but you grant MDPI AG the non-exclusive and unrevocable license right to publish this paper online on the Sciforum.net platform. This means you can easily submit your paper to any scientific journal at a later stage and transfer the copyright to its publisher (if required by that publisher). (http://sciforum.net/about ). 